\title{
Evolution of Junction Temperature and Heating Effects in UV AIGaN Nanowires LEDs
}

\author{
Davide Priante, ${ }^{1}$ Rami T. Elafandy, ${ }^{1}$ Aditya Prabaswara, ${ }^{1}$ Bilal Janjua, ${ }^{1}$ Chao Zhao, ${ }^{1}$ Malleswararao Tangi, ${ }^{1}$ \\ Mohd Sharizal Alias, ${ }^{1}$ Yazeed Alaskar, ${ }^{2}$ Abdulrahman M. Albadri, ${ }^{2}$ Ahmed Y. Alyamani, ${ }^{2}$ Tien Khee Ng, ${ }^{1}$ \\ Boon S. Ooi ${ }^{1}$ \\ ${ }^{1}$ King Abdullah University of Science and Technology (KAUST), Photonics Laboratory, Thuwal 23955-6900, Saudi Arabia \\ ${ }^{2}$ National Center for Nanotechnology, King Abdulaziz City for Science and Technology (KACST), Riyadh, 11442-6086, Saudi Arabia. \\ tienkhee.ng@kaust.edu.sa \\ boon.ooi@kaust.edu.sa
}

\begin{abstract}
We show reduced junction temperature and heat dissipation in AlGaN nanowires LEDs on a metal substrate compared to devices on a silicon substrate by employing the forward-voltage and peak-shift methods.

OCIS codes: (120.6780) Temperature; (230.5590) Quantum-well, -wire and -dot devices.
\end{abstract}

\section{Introduction}

LEDs operating at high injection current exhibit a large percentage of the electrical power conversion into heat which lowers the device efficiency, thereby affecting the optical power and the reliability[1]. The diode junction temperature $\left(\mathrm{T}_{\mathrm{j}}\right)$ plays a key role on the efficiency of LEDs. AlGaN-based nanowires (NWs) LEDs have attracted attention thanks to their strain- and dislocation-free properties, device scalability, and growth on low-cost substrates. However, AlGaN NWs LEDs show significantly reduced power output and quantum efficiency compared to the planar structures. Proper thermal measurements, such as the junction temperature, have not been performed, hence representing a research gap that needs to be addressed to overcome these problems. Here, we present the first experimental $T_{j}$ measurements on AlGaN NWs LEDs grown on metal[2] and silicon[3] substrates using the forward-voltage (FV) and peak-shift (PS) methods. We obtained reduced $\mathrm{T}_{\mathrm{j}}$ for the device on metal due to the better electrical-to-optical power conversion as well as the absence of the thermally insulating $\operatorname{Si}_{\mathrm{x}} \mathrm{N}_{\mathrm{y}}$ layer at the NWs/silicon interface. Our work is directed to enlighten the UV NWs community on addressing the $\mathrm{T}_{\mathrm{j}}$ problems for eventual high stability and high power ultraviolet NWs optoelectronic devices.

\section{Results and discussion}

The FV method is based on the Shockley diode equation[4]. Experimentally, with increasing temperature the junction voltage linearly decreases when working at constant current. Therefore the temperature coefficient $\left(K_{T}\right)$ can be extracted. Figure 1(a-b) show the forward voltage vs. heat sink temperature at different injection currents for both samples on metal and silicon substrate, respectively. By measuring the instant $\left(V_{F O}\right)$ and equilibrium voltage $\left(V_{F T}\right)$ (after $30 \mathrm{~min}$ ) we can then calculate the diode $\mathrm{T}_{\mathrm{j}}$ (equation in Fig. 1(a)).
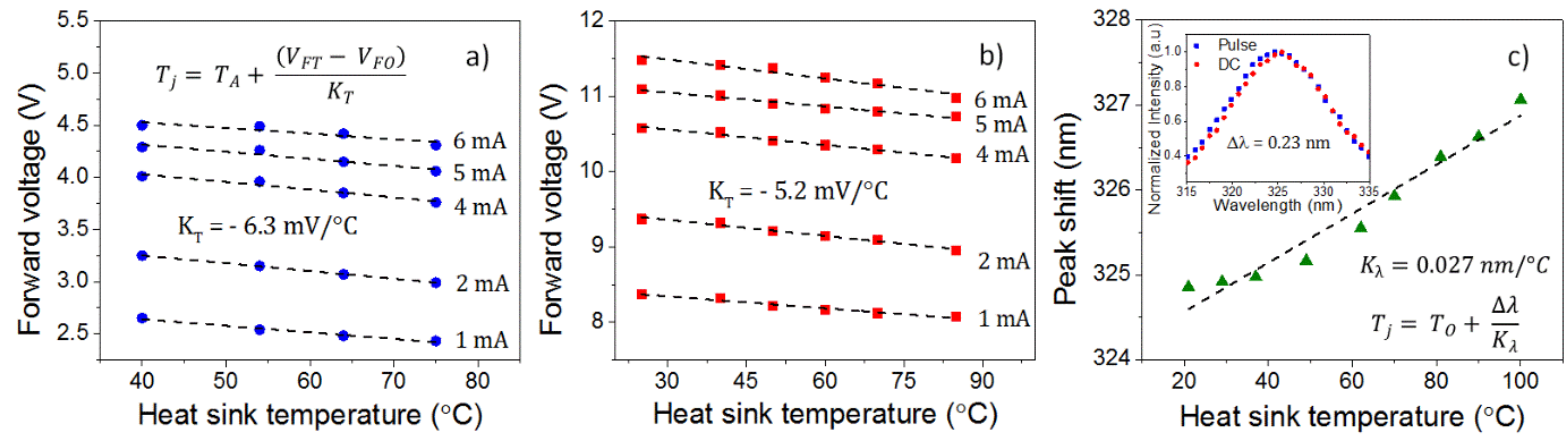

Fig. 1. Calibration measurements. (a-b) Forward-voltage vs heat sink temperature plot for the $0.5 \times 0.5 \mathrm{~mm}^{2} \mathrm{AlGaN}$ NWs on metal and silicon substrate. $T_{j}$ is the junction temperature, $T_{A}$ is the ambient temperature, $V_{F O}$ is the instant voltage and $V_{F T}$ is the equilibrium voltage. (c) Wavelength peak-shift vs heat sink temperature. The inset shows the DC and pulse spectrum difference. $\mathrm{T}_{\mathrm{O}}$ is the ambient temperature, $\Delta \lambda$ is the peak emission wavelength difference measured in DC and pulsed current. 
To compare the results obtained with the FV method, we utilized the electroluminescence (EL) PS method. Figure 1(c) shows the band-gap-dependent temperature coefficient $\left(K_{\lambda}\right)$ measurement for the sample on metal substrate. The inset shows the electroluminescence spectrum in DC and pulse mode at $100 \mathrm{~mA}$ and the equation used to calculate the $T_{j}$. The calculated $T_{j}$ is depicted in Fig. 2(a) for both samples. The NWs on metal show lower values ranging from $36-71{ }^{\circ} \mathrm{C}$ (5 to $\left.80 \mathrm{~mA}\right)$ as compared to $56-110{ }^{\circ} \mathrm{C}(5$ to $65 \mathrm{~mA})$ for the device on silicon. This can be explained by the better electrical-to-optical power conversion in the device active region on the metal substrate as well as the absence of the spontaneously formed thermally insulting $\mathrm{Si}_{\mathrm{x}} \mathrm{N}_{\mathrm{y}}$ that prevents the heat from dissipating through the silicon substrate. The EL PS method shows reduced temperatures due to the small accuracy of the EL peak that is $\sim 10 \%$ of the EL FWHM[5]. Due to the low EL intensity, the EL PS method could not be applied to the device on silicon.

Figure 2(b) shows the COMSOL simulation of the temperature as a function of the heat source density for both samples. The temperature for the sample on silicon starts deviating at $\sim 10^{14} \mathrm{~W} / \mathrm{m}^{3}$ towards higher temperatures. The inset shows the heat dissipation profile at $10^{14} \mathrm{~W} / \mathrm{m}^{3}$ for the sample on metal. Figure 2(c) shows the experimentally measured heat source density vs. current plot for both samples. At low injection currents, they have similar trends, while above $20 \mathrm{~mA}$ they start deviating due to the lower operating current for the sample on a silicon substrate. The lower heat source obtained in the sample on silicon compared to the one on metal can be explained considering that the turning point in Fig. 2(b) happens at $\sim 10^{14} \mathrm{~W} / \mathrm{m}^{3}$. However, the sample on silicon cannot reach such value (Fig. 2(c)) due to the resistive $\mathrm{Si}_{\mathrm{x}} \mathrm{N}_{\mathrm{y}}$ that hampers the current injection. We then believe that for values larger than $10^{14}$ the sample on silicon will show increased temperatures as predicted by the simulation results.
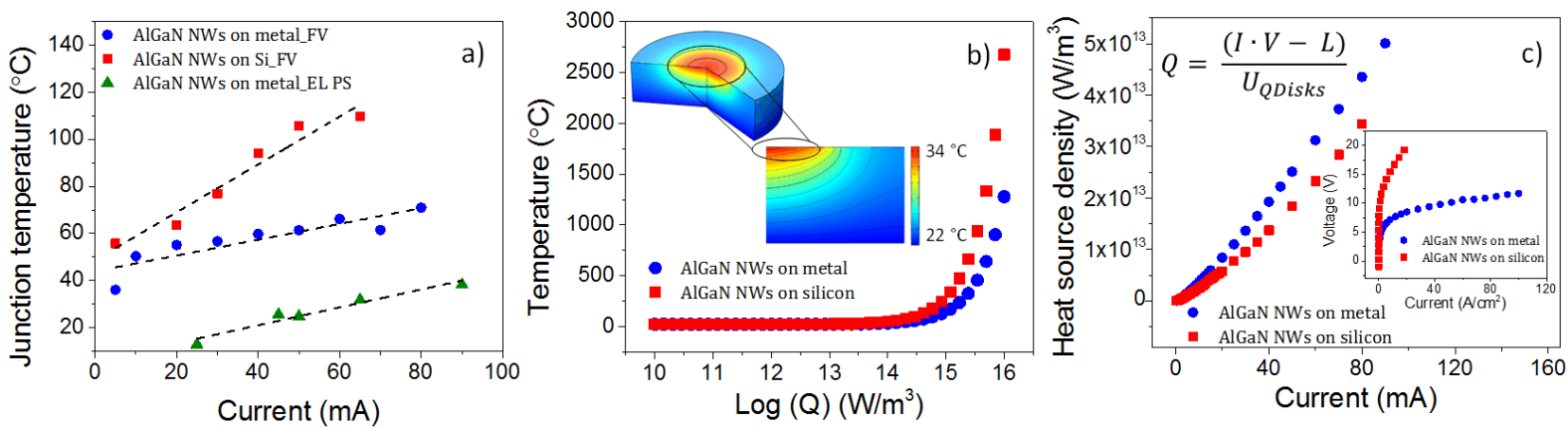

Fig. 2. (a) Junction temperature vs. injection current for the NWs LEDs on metal and silicon substrates. (b) COMSOL simulation of temperature vs. heat source density in the NWs active region. The inset shows the cross section heat dissipation for a heat source density of $10^{14} \mathrm{~W} / \mathrm{m}^{3}$. (c) Heat source density as a function of injection current. The inset shows the I-V curves.

\section{Conclusion}

The junction temperature of UV AlGaN NWs LEDs on metal thin-film and the silicon substrate is compared. We achieved lower temperatures for the NWs device on a metal substrate $\left(\sim 61^{\circ} \mathrm{C}\right.$ at $\left.50 \mathrm{~mA}\right)$ compared to the device on a silicon substrate $\left(\sim 106^{\circ} \mathrm{C}\right.$ at $\left.50 \mathrm{~mA}\right)$ by using the forward-voltage method. The peak-shift method was also adopted along with the simulation of the heat source density in the NWs active region. This study aims to advance the NWs research for achieving reliable and high power light emitters.

\section{References}

[1] T. Egawa, H. Ishikawa, T. Jimbo, and M. Umeno, "Optical degradation of InGaN/AlGaN light-emitting diode on sapphire substrate grown by metalorganic chemical vapor deposition," Applied physics letters, vol. 69, pp. 830-832, 1996.

[2] D. Priante, B. Janjua, A. Prabaswara, R. C. Subedi, R. T. Elafandy, S. Lopatin, et al., "Highly uniform ultraviolet-A quantum-confined AlGaN nanowire LEDs on metal/silicon with a TaN interlayer," Optical Materials Express, vol. 7, pp. 4214-4224, 2017.

[3] B. Janjua, H. Sun, C. Zhao, D. H. Anjum, F. Wu, A. Alhamoud, et al., "Self-planarized quantum-disks-in-nanowires ultraviolet-B emitters utilizing pendeo-epitaxy," Nanoscale, 2017.

[4] Y. Xi and E. Schubert, "Junction-temperature measurement in GaN ultraviolet light-emitting diodes using diode forward voltage method," Applied Physics Letters, vol. 85, pp. 2163-2165, 2004.

[5] Y. Xi, J.-Q. Xi, T. Gessmann, J. Shah, J. Kim, E. Schubert, et al., "Junction and carrier temperature measurements in deep-ultraviolet light-emitting diodes using three different methods," Applied physics letters, vol. 86, p. 031907, 2005.

\section{Acknowledgements}

We acknowledge the support from KACST, Grant No. KACST TIC R2-FP-008 and KAUST, BAS/1/1614-01-01. 